\title{
Engaging with African physics
}

\author{
Physics in Africa is often overlooked. We explore the challenges facing African physicists and \\ what their needs are as authors and readers.
}

An important event of 2020 has been the international rise of the Black Lives Matter movement - including within physics. This movement has amplified conversations on how colonial history continues to affect science today. One of the largest regions affected by colonialism is Africa, where $16 \%$ of the world's population lives. Despite its human potential and the fact that the continent has the youngest population on the planet, physics from Africa is under-represented in academic journals and scientific meetings. For example, none of the 25 countries/regions that published the most physics papers in 2019 is in Africa (data from Web of Science). To understand the reasons behind this under-representation, we asked ten African physicists from across the continent to share their opinion about the challenges and opportunities they face.

Unsurprisingly, their answers, published in a Viewpoint in this Issue, are diverse. They reflect the concerns of researchers at different career stages working at institutions in regions with varied cultural, social and economic environments. However, an overarching theme did emerge from their views: the uneven pace of development of physics and astronomy across Africa. There are exciting projects such as the Square Kilometre Array Telescope or the planned African Lightsource, but in other places physicists struggle with basic infrastructure needs.

Academic grievances often come down to funding. Several Viewpoint authors commented on their experiences of lacking resources for their research, and of finding that African governments undervalue science in society. Many university positions are focused on teaching, and only a few physics graduates go on to pursue research. With limited funding, research activities are often directed towards theory. Funding from agencies outside Africa is available, but runs the risk of bias towards projects that promote the agendas those agencies think are important, instead of the projects that African physicists want to pursue, as pointed out in the Viewpoint by Cameroonian physicist Dikandé Alain Moise.

Visibility in high-profile journals and at international conferences leads to international funding, collaborations and other opportunities, but African physicists are faced with several barriers. One is unconscious bias on the part of editors and referees, who may assume that work is low-quality because it has come from an under-resourced region. In addition, African researchers are under-represented at international conferences not only because of the travel costs but also, as Nigerian physicist Ibiyinka Fuwape notes, because many 'routinely' face difficulties obtaining visas. As a result, African physicists often miss opportunities to be part of the conversations taking place at scientific meetings - the very conversations that shape what the physics community thinks are the priority-defining questions it should strive to answer.

Several Viewpoint authors discussed the importance of physics for practical development in Africa: the need to develop medical physics in the continent, and the importance of physics for technologies used in agriculture, energy and water supplies. Although these are by no means the only topics of interest to African physicists, they are important, and are not in the mainstream of topics pursued by the rest of the physics community and prominently represented in high-profile physics journals.

We believe that scientific journals can break this apparent catch-22. The most obvious way is for editors to be mindful of unconscious biases when handling research from African institutions and strive to identify potential African authors for commissioned content. But journals also have a role in moderating the conversation about what the priorities of the physics community are. With regard to physics for sustainable development, there is some hope to be found in newer high-profile journals such as Nature Energy, Nature Food and Nature Sustainability. At Nature Reviews Physics we will strive to provide a more inclusive forum for the conversation about what the important questions for the physics community should be. Doing so will involve giving opportunities to African physicists to be part of the conversation, and ensuring the physics research we cover includes the topics in which they are interested. 